\title{
3 Research Square

\section{Magnetic Activated Cell-Sorting Identifies a Unique Lung Microbiome Community Associated with Disease States}

Daniel Dunlap ( $\sim$ dunlapd2@upmc.edu )

University of Pittsburgh https://orcid.org/0000-0001-9164-0748

Libing Yang

University of Pittsburgh

Shulin Qin

University of Pittsburgh

Kelvin Li

University of Pittsburgh

Adam Fitch

University of Pittsburgh

Laurence Huang

University of California San Francisco

Bryan J McVerry

University of Pittsburgh

\section{Barbara A Methé}

University of Pittsburgh

Alison Morris

University of Pittsburgh

\section{Research}

Keywords: Microbiome, HIV, host response

Posted Date: October 29th, 2021

DOI: https://doi.org/10.21203/rs.3.rs-1021798/v1

License: (c) (i) This work is licensed under a Creative Commons Attribution 4.0 International License.

Read Full License 


\section{Abstract}

Background: The advent of culture-independent, next-generation DNA sequencing has led to discovery of distinct lung bacterial communities. Studies of lung microbiome taxonomy often reveal only subtle differences between health and disease, but microbial host response may distinguish members of similar communities in different populations. Magnetic-activated cell sorting has been applied to the gut microbiome to identify numbers and types of bacteria eliciting a humoral response. We adapted this technique to examine populations of immunoglobulin-bound bacteria and investigate the lung microbiota in Human Immunodeficiency Virus (HIV) as a representative disease.

Methods: 42 people living with HIV and 22 HIV-uninfected individuals underwent bronchoalveolar lavage. We separated immunoglobulin G-bound bacteria using magnetic-activated cell sorting and sequenced the 16S rRNA gene on the Illumina MiSeq platform. We analyzed microbial sequencing data and quantified cytokines and bacterial rRNA copy numbers from bronchoalveolar lavage.

Results: Immunoglobulin G-bound bacteria were detectable in the healthy lung microbiota. Comparison of raw bronchoalveolar lavage by HIV status showed no significant taxonomic differences, but the immunoglobulin-bound lung microbiota differed by HIV status with greater abundance of Pseudomonas in people living with HIV. Lung cytokine levels were also higher in people living with HIV, which correlated with increased quantity of immunoglobulin G-bound bacteria.

Conclusions: We report a novel application of magnetic-activated cell sorting to identify immunoglobulin G-bound bacteria in the lung. This technique identified distinct bacterial communities which differed in composition from raw bronchoalveolar lavage, revealing differences in health and disease not detected by traditional analyses. Cytokine response was also associated with differential immunoglobulin binding of lung bacteria, suggesting functional importance of these communities.

\section{Background}

Studying the lung microbiome is challenging given its low biomass, the propensity to detect contaminating bacteria using next-generation sequencing techniques, and the inability to determine functional impact of pulmonary microbes $[1,2]$. As a result, studies that compare the lung microbiome in different diseases such as COPD or HIV often have conflicting conclusions or find few major differences between bacterial communities [3].

Magnetic-activated cell sorting (MACS) can be used to separate and identify immunoglobulin-bound bacteria. Bacteria are stained with fluorophores specific for bacterial DNA and the Fc region of any immunoglobulin adhered to the bacterial cell wall. Magnetic labeling of stained bacteria allows IgGbound and un-bound bacteria to be separated and analyzed. Recently, this technique has been applied to studies of the gut microbiome, and immunoglobulin (Ig)-bound bacteria have been found to modulate inflammatory bowel disease $[4,5]$. This technique has not been applied to the lung, but could serve to distinguish bacteria provoking an immune response versus those that are merely "innocent bystanders." 
We adapted the MACS technique from gut studies and applied it to the lung and bronchoalveolar lavage (BAL) in order to determine if IgG-bound bacteria differ from unsorted (raw) bacteria. We used human immunodeficiency virus (HIV) as a representative disease to evaluate differences in IgG-bound bacterial communities in the lungs of individuals with and without HIV infection. We performed quantitative polymerase chain reaction (qPCR) and next-generation sequencing on the 16S rRNA gene in IgG-bound and unsorted (raw) BAL samples. In addition, we quantified lung cytokine levels from raw BAL. We hypothesized that IgG-bound bacteria would be different from the overall bacterial communities and that people living with HIV (PLWH) would have increased recognition of and subsequent response to the lung microbiome via binding of IgG to resident bacteria.

\section{Methods}

\section{Study participants}

We recruited individuals with and without HIV infection through the University of Pittsburgh HIV research study at the Pittsburgh, Los Angeles and San Francisco sites. Subjects had no acute respiratory symptoms at the time of enrollment and were, specifically, without fevers, upper respiratory infection, or lung infection. Exclusion criteria also included those who had received systemic corticosteroids within the previous 6 months and individuals receiving antibiotic therapy within 3 months of participation.

Individuals participated in a variety of studies of pulmonary disease in HIV [6] and we included those with sufficient volumes of BAL for evaluation. All participants provided written informed consent. The University of Pittsburgh, University of California, Los Angeles, and University of California, San Francisco institutional review boards approved this study.

\section{Sample Collection}

We collected lung samples via bronchoscopy as previously described [6]. Participants were instructed to avoid any food or beverages beginning at midnight before bronchoscopy and abstain from smoking at least 12 hours prior to the procedure. All subjects gargled an antiseptic just prior to instrumentation in an attempt to limit bacteria in the oropharynx. Before bronchoscopy, 10 to $50 \mathrm{~mL} 0.9 \%$ normal saline was flushed through the bronchoscope and used as a negative control. Under moderate sedation, the bronchoscope was passed through the vocal cords and quickly placed into the wedge position in the right middle lobe. We collected BAL using sterile $0.9 \%$ normal saline to a maximal instillation volume of 200 $\mathrm{mL}$. BAL specimens were immediately fractioned into 1 and $5 \mathrm{~mL}$ aliquots and stored at

$-80^{\circ} \mathrm{C}$.

\section{Magnetic-activated cell sorting}

One $\mathrm{mL} \mathrm{BAL}$ and $1 \mathrm{~mL}$ buffer (1\% BSA in PBS) were filtered into $2 \mathrm{cc}$ Eppendorf tubes and bacteria was isolated via centrifugation (8000 RPM $\times 5$ minutes). We removed a small amount of bacteria suspended in buffer to be used in compensation controls for flow cytometry at the conclusion of each sort (described below). Remaining bacterial pellets were suspended in a solution containing the bacterial DNA stain, 
SytoBC (Thermo Fisher Scientific, Waltham, MA) and the immunoglobulin stain, IgG-PE (eBioscience, Thermo Fisher Scientific, Waltham, MA). We incubated stained bacteria with anti-PE microbeads (Miltenyi Biotec, USA) then used centrifugation to purify magnetically-labeled, stained bacteria prior to magneticactivated cell sorting (MACS).

After washing MACS columns (Miltenyi Biotec) with buffer, we poured individual bacterial samples into corresponding MACS columns that were embedded within a supermagnet (OctoMACS Separator, Miltenyi Biotec). Fifteen $\mathrm{mL}$ conicals (IgG-unbound) were stationed beneath each column. Once the entire suspended bacterial sample passed through, each column was removed from the OctoMACS Separator, placed within a different IgG-bound conical, and flushed with $1 \mathrm{~mL}$ buffer. The buffer was vigorously plunged through the column, per manufacturer's instructions, removing the magnetically-labeled material that had been held in suspension during the assay. Columns were then removed from their respective IgG+ conicals and placed atop a vacuum manifold (Promega, Madison WI) where they were washed with $70 \% \mathrm{EtOH}$ followed by buffer. Columns were replaced to their prior locations within OctoMACS Separator and the contents within the IgG-conicals were poured into the same columns now directly above. An additional washing step followed, with another $1 \mathrm{~mL}$ buffer plunged through the column into the same IgG+ conical, bringing the total volume within each conical (both IgG-bound and IgG-unbound) to $2 \mathrm{~mL}$, $450 \mu \mathrm{L}$ of which was separated and used for flow cytometry analysis.

\section{Flow cytometry}

We performed flow cytometry immediately following each MACS assay. Four hundred-fifty $\mu \mathrm{L}$ from IgGbound and IgG-unbound aliquots were stored at $4^{\circ} \mathrm{C}$ for no more than 2 hours prior to analysis. After performing stain compensation on the STI Fortessa (STI Electronics, Madison, AL), all samples were run at high throughput speed for 4 minutes. Plotting side scatter (SCC-A) against fluorescein isothiocyanate (FITC, emission $488 \mathrm{~nm}$ ) was used to gate the appropriate population of interest. A second plot of FITC vs phycoerythrin (PE, emission 578nm) was performed. Gated reads with both a positive FITC and PE signal $\left(<10^{3}\right)$ were quantified, representing the relative amount of IgG-bound bacteria in a given sample.

Negative column controls, which contained only processed, stained buffer were performed during sample runs as a quality control and used for subsequent $16 \mathrm{~S}$ control.

\section{Quantitative PCR}

Quantitative PCR amplification was performed in a total volume of $20 \mu \mathrm{L}$ amplification reaction consisting of $2 \mu \mathrm{L}$ of $10 X P C R$ buffer, $3.5 \mathrm{mmol} / \mathrm{L} \mathrm{MgCl} 2,0.2 \mathrm{mmol} / \mathrm{L}$ deoxynucleoside triphosphate, 0.5 $\mu \mathrm{mol} / \mathrm{L}$ forward and reverse primers, $0.225 \mu \mathrm{mol} / \mathrm{L}$ probe, $0.75 \mathrm{U}$ of Platinum Taq polymerase (Invitrogen), and $2 \mu \mathrm{L}$ of each DNA. The forward and reverse primers were used to amplify DNA templates encoding 16S rRNA and both primer and probe sequences were identical to those previously described [7]. A standard curve was created from serial dilutions of plasmid DNA containing known copy numbers of the template. The assays were performed on the LightCyler System (Roche) using the following PCR conditions: $95^{\circ} \mathrm{C}$ for $5 \mathrm{~min}$, followed by 50 cycles at $95^{\circ} \mathrm{C}$ for $15 \mathrm{~s}$ and at $60^{\circ} \mathrm{C}$ for $1 \mathrm{~min}$.

\section{Luminex panels and cytokine analysis}


We used a commercially available Luminex assay (Bio-Plex Pro ${ }^{\text {TM }}$ Human Cytokine 17-plex Assay, Bio-Rad Laboratories, Hercules CA) to quantify cytokines present within BAL, per manufacturer's instructions. The cytokines used for analysis included interleukin (IL)-1ß, IL-2, IL-6, IL-8, macrophage inflammatory protein (MIP-1B, also known as CCL4), interferon (IFN)-y, and tumor necrosis factor (TNF)-a. No dilutions were performed.

\section{Sample processing and sequencing}

We extracted bacterial DNA from both sorted and unsorted BAL samples using DNeasy PowerSoil Kits (Qiagen, Germantown MD) per manufacturer's instructions, then amplified the V4 subunit of the bacterial $16 \mathrm{~S}$ gene using polymerase chain reaction (PCR). We performed sequencing on the Illumina MiSeq platform [8] and de-multiplexed reads onboard the machine using standard Illumina software. We then performed post-sequencing quality control (including sequence filtering and trimming raw 16S sequence) using an in-house pipeline developed by the University of Pittsburgh Center for Medicine and the Microbiome, utilizing available software including fastx toolkit, cutadapt, and dust [9-11]. Contamination controls were performed at every step of the process. Distribution-based contaminant filtering was applied to column controls to remove background signal from MACS columns and beads (Supplement 1). We generated operational taxonomic units (OTUs) and RDP classified sequences using an in-house mothur pipeline $[12,13]$.

\section{Quantifying immunoglobulin levels}

Both serum and BAL immunoglobulin G levels were quantified. Serum samples were sent to the University of Pittsburgh Medical Center Clinical Laboratory (3460 Fifth Ave, Pittsburgh, PA), who quantified levels using commercially available ELISA assays. To quantify BAL IgG levels, we used a commercially available ELISA assay (Human IgG ELISA Kit, Abcam, Cambridge, MA) using manufacturer's instructions, diluting BAL at a 1:500 ratio.

\section{Statistical analysis}

We applied a non-metric multidimensional scaling plot (NMDS) using the Bray-Curtis dissimilarity index to visualize sample clustering and performed multivariate analysis of variance (PERMANOVA) to compare the microbiota by HIV status, IgG status, ART status, and BAL cytokine level with the $R$ vegan package. We performed paired testing of BAL samples between IgG-bound and unsorted samples using Wilcoxon paired p-values to detect differences in abundance of individual bacterial genre. Non-parametric t-testing (Mann U Whitney) was used to compare groups by HIV status and use of ART. Cytokine levels were log-transformed and plotted against log-transformed IgG-bound bacteria and qPCR data, and a linear regression model was used to evaluate correlation between the two variables.

\section{Results}

Twenty-two HIV-uninfected participants and 42 PLWH were included. Age was similar between groups while gender, race, and smoking status differed (Table 1). Sixty-four IgG-bound, IgG-unbound, and raw 
samples were analyzed, though one raw sample had zero reads and was excluded from subsequent $16 \mathrm{~S}$ analysis. IgG-unbound sorted aliquots largely resembled column contamination and were thus excluded from subsequent analysis.

Table 1

Participant Demographics by HIV Status ( $\mathrm{n}=64)$

\begin{tabular}{|lll|}
\hline & PLWH & HIV-uninfected \\
\hline & $\mathrm{n}=42$ & $\mathrm{n}=22$ \\
\hline Age, mean (range) & $51.6(29-67)$ & $50.7(37-64)$ \\
Rale, $\mathrm{n}(\%)$ & $32(76.1)$ & $15(68.2)$ \\
Blace, $\mathrm{n}(\%)$ White & $13(30.9)$ & $12(54.5)$ \\
Other & $28(64.3)$ & $7(31.8)$ \\
\hline COPD, n (\%) & $2(4.8)$ & $3(13.6)$ \\
\hline Ever smoker, $\mathrm{n}(\%)$ & $31(73.8)$ & $8(36.3)$ \\
\hline Pack-years, median (range) & $14(0-48)$ & $6.2(0-54)$ \\
\hline CD4 count, median (range) & $694(43-1505)$ & N/A \\
\hline Receiving ART, $\mathrm{n}$ (\%) & $29(69.0)$ & N/A \\
\hline
\end{tabular}

\section{Comparison of IgG-bound lung microbiota to raw BAL microbiota in healthy, HIV-uninfected individuals}

The relative abundance and overall community structure differed in IgG-bound and unsorted samples in HIV-uninfected individuals (PERMANOVA for Bray-Curtis distance, $p<0.0001, R^{2}=0.09$; Figure $1 A$ ). While similar bacteria were seen in both raw and IgG-bound BAL samples, their relative abundance differed (Figure 1B). Streptococcus, Prevotella, and Veillonella were more abundant in raw BAL samples relative to IgG-bound BAL. These oral microbes were also seen in IgG-bound samples, but Pseudomonas was detected in greater relative abundance when compared to raw BAL samples.

\section{Comparison of raw and IgG-bound BAL by HIV status}

We then compared lung bacterial communities by HIV status and participant use of ART using flow cytometry data, $16 \mathrm{~S}$ results, and qPCR. Based on flow cytometry, PLWH had more IgG-bound bacteria in the lungs than HIV-uninfected individuals ( $p=0.0008$, Figure 2A). Further, PLWH not on ART tended to have a higher quantity of IgG-bound bacteria than those on ART. PLWH not on ART had greater IgG-bound bacteria in BAL than persons without HIV ( $p<0.0001$, Figure $2 B)$. Utilizing qPCR to quantify the number of 
bacterial 16S rRNA copies in IgG-bound BAL samples, PLWH tended to have higher IgG-bound rRNA copy numbers than HIV-uninfected individuals ( $p=0.06$, Figure $2 \mathrm{C})$. We also quantified serum and BAL IgG concentration. There was no difference in IgG concentration in PLWH compared to HIV un-infected individuals, but PLWH had high serum IgG level $(p=0.03$, Supplement 2$)$.

Next, we compared BAL microbial composition in PLWH and HIV-uninfected individuals. Similar to previous studies [3], we found no significant differences in the lung microbiota when comparing PLWH to HIV-uninfected individuals (Figure 3A). However, when we performed next-generation sequencing on IgGbound samples, bacterial communities stratified by HIV status (PERMANOVA for Bray-Curtis distance, $\mathrm{p}=0.008, \mathrm{R}^{2}=0.03$; Figure $3 \mathrm{~A}$ ). In PLWH, Tropheryma, Veillonella, and Prevotella were the most abundant bacteria in raw BAL samples. In contrast, Pseudomonas was by far the most abundant IgG-bound bacteria in PLWH. In both HIV-uninfected individuals and PLWH, Pseudomonas was relatively more abundant in IgG-bound BAL samples when compared to raw but was significantly more abundant in PLWH (Figure 3B).

\section{Relationship between lung cytokine levels and IgG-bound bacteria in HIV}

To further explore an association between host recognition of the lung microbiome and inflammatory response, we quantified BAL cytokine levels in the PLWH and linked these to levels of IgG-bound bacteria. We found that PLWH had significantly higher levels of BAL cytokines including IL-8, IFN-y, MCP-1 and TNF- $\alpha$ as previously described [14]. IL- 6 and IL-1 $\beta$ levels also tended to be higher in PLWH (Supplement 3). In PLWH, higher levels of IgG-bound bacteria were associated elevated BAL cytokine levels including IL-8 and IL-1 $\beta$ by $q P C R\left(p=0.003, R^{2}=0.2\right.$ and $p=0.012, R^{2}=0.15$, respectively) and flow cytometry $\left(p=0.008, R^{2}=0.16\right.$ and $p<0.001, R^{2}=0.32$, respectively; Figure 4$)$. There was no correlation between IL-8 and IL-1 $\beta$ cytokine levels with IgG-bound bacteria in HIV-uninfected individuals.

\section{Discussion}

Using a novel application of magnetic-activated cell sorting to isolate lgG-bound bacteria and stufy the lung, we identified a distinct immunoglobulin-bound lung microbiota. Microbes bound by IgG were present in different relative abundance, resulting in markedly different community structure. Despite relatively high abundance of Tropheryma and oral anaerobes in unsorted samples, Pseudomonas was the most abundant bacteria recognized and bound by IgG. Using this technique, we detected differences in the lung microbiota in HIV infection that were not apparent when performing typical 16S rRNA gene sequencing of whole BAL. Further, IgG-bound bacteria in HIV were associated with increased inflammation within the lungs. Application of this technique may expand investigation of the lung microbiome in health and disease.

Initial work with MACS has been applied to study bacteria in the gut and gastrointestinal disorders. For example, using a murine model, researchers demonstrated that IgA-bound bacteria resulted in increased 
susceptibility to colitis in germ-free mice [4]. In a subsequent human study, IgG binding identified bacteria implicated in inflammatory bowel disease pathogenesis in pediatric patients [5].

In the first investigation applying the MACS technique to the lung, we were able to isolate and quantify IgG-bound bacteria and reliably detect a different bacterial community structure than revealed by $16 \mathrm{~S}$ rRNA gene sequencing of whole BAL. Although the typical oral bacteria identified in the lung microbiome were seen in both IgG-bound and raw BAL, there was relatively greater IgG-binding of Pseudomonas. Functional significance of immunoglobulin-bound bacteria is not known, but suggests differences in host response to similar bacteria and may contribute to previously defined "pneumotypes" [15].

We investigated HIV as a representative condition for applying this technique to the study of pulmonary disease, and we were able to detect clear differences that were not seen in analyses of whole BAL. Prior investigation of the lung microbiome in HIV infection has failed to find significant taxonomic differences in individuals with normal CD4 counts on appropriate antiretroviral therapy [1,3]; a somewhat unexpected finding given the differences in lung immune responses and pulmonary diseases in this population [1620]. PLWH had greater abundance of IgG-bound respiratory pathogens such as Pseudomonas and Stenotrophomonas. Elevated levels of multiple cytokines, including IL- 6 and IL-1 $\beta$, were correlated with higher numbers of IgG-bound bacteria. This correlation suggests that host recognition of the lung microbiota may stimulate chronic lung inflammation. It is possible that HIV-infected individuals have increased recognition of the lung microbiota via binding of IgG to resident bacteria, leading to an increased inflammatory response and ultimately contributing to chronic pulmonary disease seen in this population $[16,18,20]$. Future studies will be useful to examine this link.

Interestingly, Tropheryma was identified in raw BAL samples in both PLWH and healthy controls, but it was not as abundant in the IgG-bound communities. This bacteria has been consistently detected in the lung in both healthy populations and in PLWH [21]. It has been associated with smoking and its prevalence decreases with initiation of ART. Despite its presence in the lung, it has not been associated with pulmonary inflammation or lung function [22]. The discordance between its high abundance in raw samples and relatively lower abundance in IgG-bound samples could suggest that the absence of an inflammatory response to Tropheryma in the lungs may be due to a lack of active host recognition and response [21, 22].

It is plausible that immunoglobulin-bound bacteria play a role in lung disease. Though traditionally considered protective, immunoglobulin binding of bacteria can lead to inflammation through opsonization [23] and antibody-dependent cell-mediated cytotoxicity [24]. B cells have been identified in greater abundance from the lungs of individuals with severe COPD [25] and have been implicated in emphysema pathogenesis [26, 27]. Host recognition of the lung microbiome corresponds to an inflammatory response [15]. Our results suggest that one possible mechanism of host recognition and response to the microbiome is through the adaptive immune system. Further, the presence of IgG-bound bacteria in the lungs given the relative absence of IgG in the mouth could suggest an alternative mode of bacterial migration into the lungs or recognition of unbound bacteria in the lungs. 
Our study has several limitations. Given the low biomass within the lungs, any degree of experimental contamination can significantly skew results. We attempted to combat the impact of contamination by filtering all media, running UV light in the biosafety cabinet at the beginning and conclusion of each assay, and performing controls at each step of our experiment. We ran MACS controls, where $1 \%$ buffer was stained and run through the columns and subsequently sequenced. Using the sequencing data from the columns, we were able to subtract the $16 \mathrm{~S}$ signal attributable to column contamination, reducing the amount of background noise. Additionally, controls were performed during each step of the PCR process. We examined only IgG, but IgM or IgA binding might identify other bacterial communities of importance. We chose IgG as our initial analysis based on preliminary fluorescence-activated cell sorting data demonstrating the strongest signal (data not shown). Other variables such as smoking or prior lung infections could also impact results.

In conclusion, we report the first study of the immunoglobulin-bound lung microbiome. Using this technique, we identified distinct bacterial communities in the healthy lung and in HIV-infected individuals. IgG-bound bacteria showed greater differences by HIV status than seen in traditional analyses of BAL, and the relationship to inflammation suggests a potential role in disease. Though we chose to study immunoglobulin binding in HIV, adaptive immunity has been implicated in myriad chronic pulmonary diseases $[25,28,29]$, thus study of host recognition and response to the lung microbiome can be applied to investigation of other lung diseases, and modulation of immunoglobulin-binding of the lung and oral microbiome could be a therapeutic target.

\section{Declarations}

Ethics approval and consent to participate

The University of Pittsburgh IRB reviewed and approved these studies. The reference numbers for these studies are:

TEAL - STUDY19070181

LEAP - STUDY19080258

Consent for publication

Not applicable.

Availability of data and material

Data sets generated and analyzed during the current study are available in the NIH Sequence Read Archive (SRA) Sequencing data was uploaded to the NIH Sequence Read Archive (SRA), PRJNA720126 [https://www.ncbi.nlm.nih.gov/sra/?term=PRJNA720126]

Competing interests 
The authors declare that they have no competing interests.

Funding

Provided by the National Institutes for Health: NIH R01HL120398, K24HL123342, R01HL125049 (AM); K24HL087713 (LH); University of Pittsburgh CTSI (UL1 TR000005); UL1 TR000004 (UCSF CTSA); 5R21HL143091 (BAM).

Authors' contributions

All authors were involved with editing the manuscript. DGD performed the bulk of bench work, wrote the manuscript, and participated in data analysis. YL assisted with DNA exstraction, PCR, and data analysis. SQ performed $\mathrm{qPCR}$. $\mathrm{KL}, \mathrm{AF}$, and $\mathrm{BM}$ applied sequencing data to pipeline and analyzed microbiome data. $\mathrm{LH}$ and BM contributed to study design and assisted with manuscript development. AM oversaw preliminary experiments which contributed to study design and was involved with data analysis, manuscript preparation, and sample collection. All authors read and approved the final manuscript.

Acknowledgements

Not applicable.

\section{References}

1. Beck JM, Schloss PD, Venkataraman A, Twigg H, Jablonski KA, Bushman FD, et al. Multicenter comparison of lung and oral microbiomes of HIV-infected and HIV-uninfected individuals. Am J Respir Crit Care Med. 2015;192:1335-44. doi:10.1164/rccm.201501-01280C.

2. Bassis CM, Erb-Downward JR, Dickson RP, Freeman CM, Schmidt TM, Young VB, et al. Analysis of the upper respiratory tract microbiotas as the source of the lung and gastric microbiotas in healthy individuals. MBio. 2015;6:e00037. doi:10.1128/mBio.00037-15.

3. Cribbs SK, Uppal K, Li S, Jones DP, Huang L, Tipton L, et al. Correlation of the lung microbiota with metabolic profiles in bronchoalveolar lavage fluid in HIV infection. Microbiome. 2016;4:3. doi:10.1186/s40168-016-0147-4.

4. Palm NW, de Zoete MR, Cullen TW, Barry NA, Stefanowski J, Hao L, et al. Immunoglobulin A coating identifies colitogenic bacteria in inflammatory bowel disease. Cell. 2014;158:1000-10. doi:10.1016/j.cell.2014.08.006.

5. Armstrong $\mathrm{H}$, Alipour $\mathrm{M}$, Valcheva R, Bording-Jorgensen $\mathrm{M}$, Jovel J, Zaidi D, et al. Host immunoglobulin $\mathrm{G}$ selectively identifies pathobionts in pediatric inflammatory bowel diseases. Microbiome. 2019;7:1. doi:10.1186/s40168-018-0604-3.

6. Morris A, Beck JM, Schloss PD, Campbell TB, Crothers K, Curtis JL, et al. Comparison of the respiratory microbiome in healthy nonsmokers and smokers. Am J Respir Crit Care Med. 2013;187:1067-75. doi:10.1164/rccm.201210-19130C. 
7. Liu CM, Aziz M, Kachur S, Hsueh P-R, Huang Y-T, Keim P, et al. BactQuant: an enhanced broadcoverage bacterial quantitative real-time PCR assay. BMC Microbiol. 2012;12:56. doi:10.1186/14712180-12-56.

8. Caporaso JG, Lauber CL, Walters WA, Berg-Lyons D, Huntley J, Fierer N, et al. Ultra-high-throughput microbial community analysis on the Illumina HiSeq and MiSeq platforms. ISME J. 2012;6:1621-4. doi:10.1038/ismej.2012.8.

9. Morgulis A, Gertz EM, Schäffer AA, Agarwala R. A fast and symmetric DUST implementation to mask low-complexity DNA sequences. J Comput Biol. 2006;13:1028-40. doi:10.1089/cmb.2006.13.1028.

10. Blankenberg D, Gordon A, Von Kuster G, Coraor N, Taylor J, Nekrutenko A, et al. Manipulation of FASTQ data with Galaxy. Bioinformatics. 2010;26:1783-5. doi:10.1093/bioinformatics/btq281.

11. Martin M. Cutadapt removes adapter sequences from high-throughput sequencing reads. EMBnet $j$. 2011;17:10. doi:10.14806/ej.17.1.200.

12. Schloss PD, Westcott SL, Ryabin T, Hall JR, Hartmann M, Hollister EB, et al. Introducing mothur: opensource, platform-independent, community-supported software for describing and comparing microbial communities. Appl Environ Microbiol. 2009;75:7537-41. doi:10.1128/AEM.01541-09.

13. Wang Q, Garrity GM, Tiedje JM, Cole JR. Naive Bayesian classifier for rapid assignment of rRNA sequences into the new bacterial taxonomy. Appl Environ Microbiol. 2007;73:5261-7. doi:10.1128/AEM.00062-07.

14. Twigg HL. Bronchoalveolar lavage fluid in HIV-infected patients. "Cytokine soup”. Chest. 1993;104:659-61.

15. Segal LN, Clemente JC, Tsay J-CJ, Koralov SB, Keller BC, Wu BG, et al. Enrichment of the lung microbiome with oral taxa is associated with lung inflammation of a Th17 phenotype. Nat Microbiol. 2016;1:16031. doi:10.1038/nmicrobiol.2016.31.

16. Crothers K, McGinnis K, Kleerup E, Wongtrakool C, Hoo GS, Kim J, et al. HIV infection is associated with reduced pulmonary diffusing capacity. J Acquir Immune Defic Syndr. 2013;64:271-8. doi:10.1097/QAl.0b013e3182a9215a.

17. Bigna JJ, Kenne AM, Asangbeh SL, Sibetcheu AT. Prevalence of chronic obstructive pulmonary disease in the global population with HIV: a systematic review and meta-analysis. Lancet Glob Health. 2018;6:e193-202. doi:10.1016/S2214-109X(17)30451-5.

18. Singhvi D, Bon J, Morris A. Obstructive Lung Disease in HIV-Phenotypes and Pathogenesis. Curr HIV/AIDS Rep. 2019;16:359-69. doi:10.1007/s11904-019-00456-3.

19. Risso K, Guillouet-de-Salvador F, Valerio L, Puglièse P, Naqvi A, Durant J, et al. COPD in HIV-infected patients: CD4 cell count highly correlated. PLoS One. 2017;12:e0169359. doi:10.1371/journal.pone.0169359.

20. Gingo MR, George MP, Kessinger CJ, Lucht L, Rissler B, Weinman R, et al. Pulmonary function abnormalities in HIV-infected patients during the current antiretroviral therapy era. Am J Respir Crit Care Med. 2010;182:790-6. doi:10.1164/rccm.200912-18580C. 
21. Qin S, Clausen E, Lucht L, Michael H, Beck JM, Curtis JL, et al. Presence of Tropheryma whipplei in Different Body Sites in a Cohort of Healthy Subjects. Am J Respir Crit Care Med. 2016;194:243-5. doi:10.1164/rccm.201601-0162LE.

22. Qin S, Clausen E, Nouraie SM, Kingsley L, McMahon D, Kleerup E, et al. Tropheryma whipplei colonization in HIV-infected individuals is not associated with lung function or inflammation. PLoS One. 2018;13:e0205065. doi:10.1371/journal.pone.0205065.

23. Actor JK. The Inflammatory Response. In: Introductory Immunology. 1st edition. Cambridge, MA: Academic Press; 2014. p. 16-27.

24. Mak TW, Saunders ME. B Cell Receptor Structure and Effector Function. In: The Immune Response. Cambridge, MA: Academic Press; 2006. p. 93-120.

25. Hogg JC, Chu F, Utokaparch S, Woods R, Elliott WM, Buzatu L, et al. The nature of small-airway obstruction in chronic obstructive pulmonary disease. N Engl J Med. 2004;350:2645-53. doi:10.1056/NEJMoa032158.

26. Faner R, Cruz T, Casserras T, López-Giraldo A, Noell G, Coca I, et al. Network Analysis of Lung Transcriptomics Reveals a Distinct B-Cell Signature in Emphysema. Am J Respir Crit Care Med. 2016;193:1242-53. doi:10.1164/rccm.201507-13110C.

27. Sullivan J-L, Bagevalu B, Glass C, Sholl L, Kraft M, Martinez FD, et al. B Cell-Adaptive Immune Profile in Emphysema-Predominant Chronic Obstructive Pulmonary Disease. Am J Respir Crit Care Med. 2019;200:1434-9. doi:10.1164/rccm.201903-0632LE.

28. Boyton RJ. Regulation of immunity in bronchiectasis. Med Mycol. 2009;47 Suppl 1:S175-82. doi:10.1080/13693780802163370.

29. Holgate ST. Innate and adaptive immune responses in asthma. Nat Med. 2012;18:673-83. doi:10.1038/nm.2731.

\section{Figures}



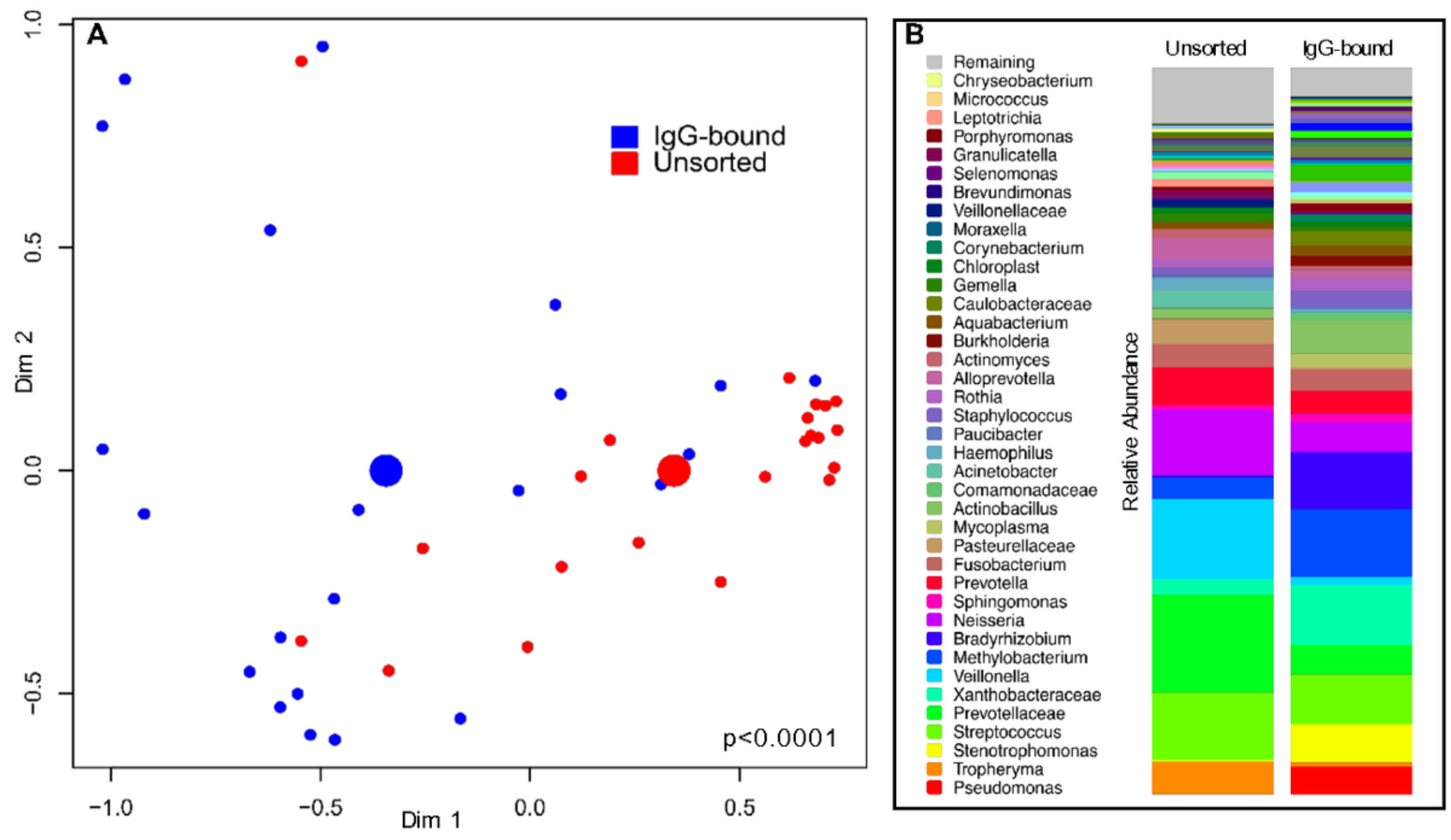

\section{Figure 1}

MACS identifies an IgG-bound lung microbiome that differs from raw BAL in healthy individuals. A nonmetric multi-dimensional scaling plot (NMDS) (A) and stacked bar plots (B) detail the differences observed between the IgG-bound and raw lung microbiome in $22 \mathrm{HIV-uninfected} \mathrm{individuals.} \mathrm{A)} \mathrm{We}$ applied an NMDS plot using Bray-Curtis dissimilarity index to visualize sample clustering and performed multivariate analysis of variance (PERMANOVA) to compare beta diversity between IgG-bound and raw (unsorted) samples from $16 \mathrm{~S}$ gene sequencing data. Points represent bacterial communities of individual samples, color-coded by IgG status (blue=lgG-bound, red=unsorted/raw). Larger colored centroids represent the mean group values. We found significant taxonomic differences between groups $(p<0.0001$, $\mathrm{R} 2=0.09$ ). B) Stacked bar plots depict the relative abundance of bacteria in unsorted and IgG-bound BAL samples from HIV-uninfected individuals. In raw BAL samples, common oral microbes such as Streptococcus, Prevotella, and Veillonella were most abundant. Relative abundance of bacteria differed in the IgG-bound fraction. 

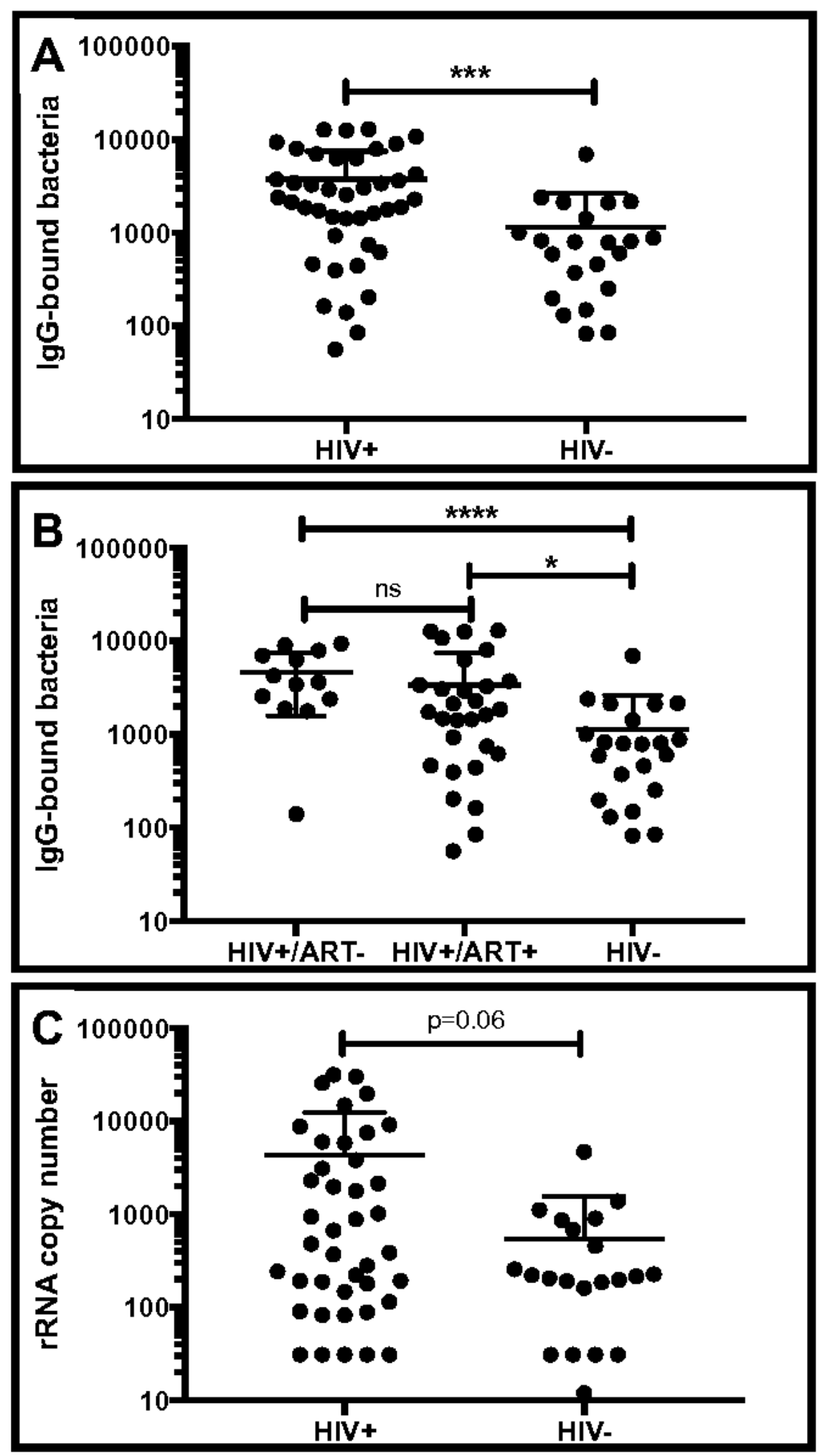

Figure 2

PLWH had greater number of IgG-bound bacteria in the lungs than HIV un-infected individuals. Study participants were grouped by HIV status and then by use of anti-retroviral therapy (ART). A) Individuals were grouped by HIV status and groups compared using non-parametric t-testing (Mann U Whitney). PLWH had significantly more IgG-bound bacteria than HIV-uninfected individuals $(p=0.0008)$. B) PLWH were then sub-divided by use of ART and compared with HIV-uninfected individuals. The three groups 
were compared using non-parametric t-tests (Mann U Whitney). PLWH not receiving ART had the highest abundance of IgG-bound bacteria by flow cytometry, when compared to HIV-uninfected individuals $(p<0.0001)$ and PLWH taking ART $(p=0.06)$. PLWH on ART also had greater abundance of IgG-bound bacteria $(p=0.017)$. C) Quantitative PCR was used to quantify rRNA copy number in IgG-bound BAL samples. PLWH tended to have higher rRNA copy number $(p=0.06)$.
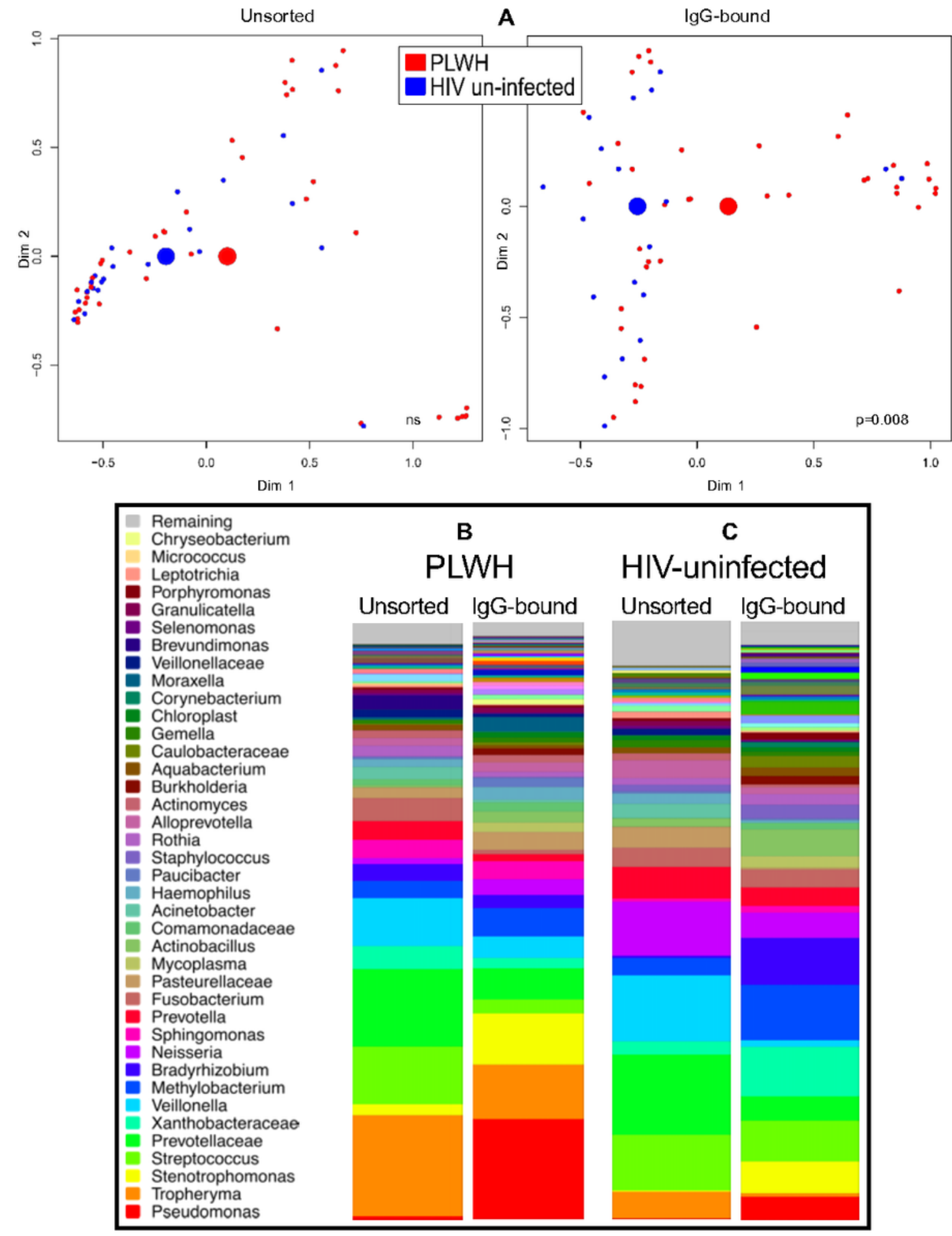

Figure 3 
Bacterial taxa stratify by HIV status in IgG-bound BAL. A) Unsorted (left) and IgG-bound (right) NMDS plots are displayed side by side to compare the differences in beta diversity observed when individuals are grouped by HIV status. We used Bray-Curtis dissimilarity index to visualize sample clustering and performed multivariate analysis of variance (PERMANOVA) to compare bacterial communities in PLWH and HIV-uninfected individuals in both unsorted (raw) and IgG-bound BAL samples. Smaller dots signify the microbial community of individual samples, while the larger centroids represent the statistical average in each grouping (red=PLWH, blue=HIV un-infected). There were no taxonomic differences between PLWH and HIV- uninfected individuals in unsorted samples; however, bacterial communities stratified by HIV status in IgG-bound BAL samples $(p=0.008)$. B) Stacked bar plots depict the relative abundance of bacteria in unsorted and IgG-bound BAL samples in PLWH. Pseudomonas was seen in greater abundance in IgG-bound samples when compared to unsorted samples. C) Stacked bar plots depict the relative abundance of bacteria in unsorted and IgG-bound BAL samples in HIV-uninfected individuals.

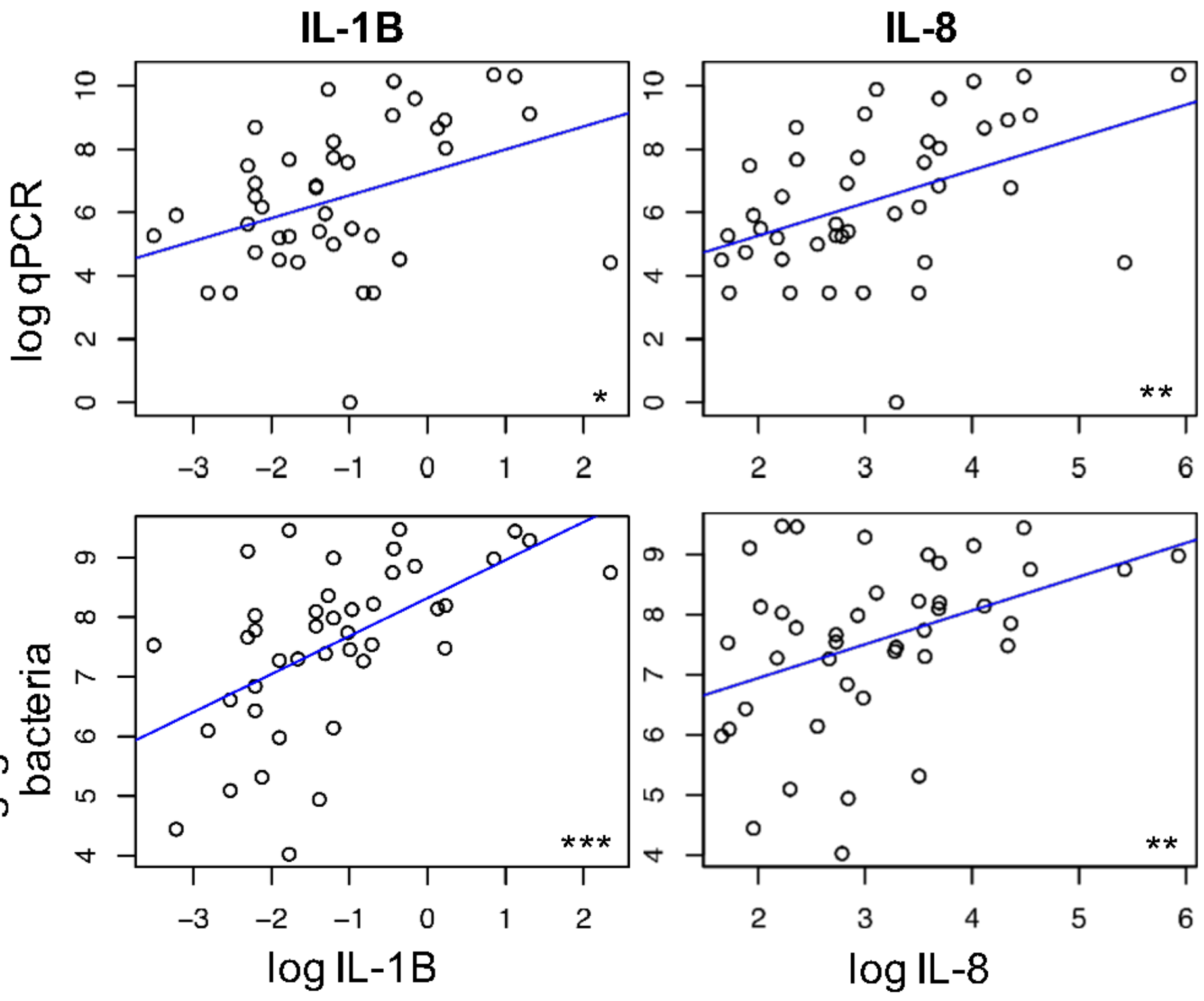

Figure 4 
Increased IgG-bound bacteria in the lungs of PLWH correlate with BAL cytokine levels. We plotted IgGbound bacteria as determined by qPCR (top) and flow cytometry (bottom) against BAL cytokine levels in PLWH. Individual dots represent the intercept between log-transformed cytokine level (x-axis) and logtransformed IgG bound bacteria quantity ( $y$-axis). A linear regression model was applied to assist with visualization of the trend and quantify the degree of association. IL-8 and IL-1 $\beta$ correlated with increasing quantity of IgG-bound bacteria by $\mathrm{qPCR}(\mathrm{p}=0.003, \mathrm{R} 2=0.2$ and $p=0.012, R 2=0.15$, respectively) and flow cytometry $(p=0.008, R 2=0.16$ and $p<0.001, R 2=0.32$, respectively $)$.

\section{Supplementary Files}

This is a list of supplementary files associated with this preprint. Click to download.

- MACSSupplementMicrobiome.docx 